\title{
XII. Zum Rheinischen Bund von 1254 .
}

Von

\author{
Dr. Julius Weizsãcker, \\ Professor in Gottingen.
}

\begin{abstract}
Der Rheinische Bund von 1254 ist in neuerer Zeit Gegenstand näherer Untersuchung gewesen. Als die einzige erfreuliche Erscheinung in den trüben Tagen des sogenannten Interregnums wird er immer wieder die Aufmerksamkeit auf sich ziehen. Aber man hat früher seine Bedeutung gern überschätzt, und es ist das unbestreitbare Verdienst Busson's ') die hohen Vorstellungen davon wieder herabgedrückt $\mathrm{zu}$ haben: der Landfriedensbund hat eine vortreffliche Idee schlecht ausgeführt, seine Organisation war nicht fest genug und ermangelte einer krāftigen Exekutive, seine Wirksamkeit war von sehr kurzer Dauer. Dagegen kann ich Busson's kritische Auffassung der uns noch erhaltenen Bundesakten keineswegs theilen, sofern der Werth eines Theils derselben ebenfalls herabgesetzt wird wegen mangelhafter Ueberlieferung, wodurch denn seine historische Verwendbarkeit leidet. Diese Akten, wie sie bei Leibnitz ${ }^{2}$ ), Freyberg ${ }^{3}$ ), Böhmer ${ }^{4}$ ), Pertz ${ }^{5}$ ), Schaab ${ }^{6}$ ) mehr oder minder gut wieder-
\end{abstract}

1) Zur Geschichte des grossen Landfriedensbundes deutscher Stådte 1254, nsbruck 1874.

$\left.{ }^{2}\right)$ Cod. jur. gent., mantissa, 2, 93-98, Hannoverae 1700.

3) Sammlung hist. Schriften und Urkunden, 1, 513-520, Stuttgart und Tübingen 1827 .

4) Cod. dipl. Moenofrancofurt. 103-113, Frankf. a. M. 1836.

5) M. G. LL. 2, 368-380, vermischt mit andern Stūcken.

$\left.{ }^{6}\right)$ Gesch. des grossen Rheinischen Stãdtebundes 2, 16-47, ebenfalls vermischt. 
gegeben sind, haben aber für uns schon in Allgemeinen eine hohe Bedeutung, weil uns hier zum erstenmal eine ganze Reihe von derartigen Bundesabschieden in ununterbrochener Folge erhalten ist, und es wăre sehr Schade, wenn sie uns durch die Form ihrer Ueberlieferung theilweise so unbrauchbar oder unsicher geworden wären, wie Busson glaubt. Sind sie zuverlāssig, so geben sie uns über die Entwicklungsgeschichte dieses eigenthümlichen Bundes höchst erwünschte und ausführliche Auskunft. Man hat früher an ihrer Authenticitāt nicht gezweifelt. Bedenken lassen sich dabei freilich nicht unterdrūcken. Aber es frăgt sich, ob man so weit gehen muss, eine ganze Reihe derselben für blosse Entwürfe oder Prāliminarpunktationen zu erklären, wie Busson gethan hat. An und für sịch ist es freilich nicht undenkbar, dass, nachdem sich so und so viele Andere mit einer Frage beschäftigt haben, der Letzte klüger ist als sie Alle. Aber dass uns Busson auch die Authenticität der Gründungsurkunde vom Juli 1254 nehmen wollte, das hat mich doch nicht schlafen lassen. Ich kam da von einer Untersuchung auf die andere, und schliesslich glaubte ich darin noch klüger geworden zu sein als Busson. Und da es mir nicht möglich war, dieses an sich schon wohlthuende Gefühl in meiner Brust zu verschliessen, so entstand daraus ein ganzes Buch über die Sache 1). Am Ende kommt gar noch Einer, der sich für klüger hălt als uns Beide zusammen. Vielleicht eine jüngere Dissertationenkraft.

Busson hat sich aber ein grosses Verdienst erworben, das ihm nicht leicht Einer wird nehmen können. Er ist es gewesen, der zuerst erkannte, dass wir die Abschiede hier nicht in ihrer authentischen Form vor uns haben. Bei den meisten derselben halte ich das für vollkommen sicher. Aber es blieben dann noch zwei Möglichkeiten. Entweder ging man hinter die endgiltige Ausfertigung zurück und erklärte das Vorhandene für Entwürfe und Präliminarpunktationen, oder man schritt über die endgiltige Ausfertigung hinaus und erklärte das Vorhandene für eine nachträgliche Bearbeitung. Jenen Weg hat Busson eingeschlagen, ich den letzteren. Dem Erfolg nach ist mein Verfahren das konservativere. Auf Busson's Weg wird die historische Verwendbarkeit der Bundesakten sehr eingeschränkt; mein Verfahren, wenn es, wie ich glaube, das richtige

1) Der Kheinische Bund 1254, Tübingen 1879. 
ist, stellt dieselbe in weitem Umfang wieder her. Ich muss zugeben, dass wir in unsern Bundesakten allerdings die Abschiede nicht in ihrer ursprünglichen Form erhalten haben, sondern mehr oder weniger verändert, abgekürzt. Aber wir haben Grund anzunehmen, dass uns von denselben nichts wesertliches verloren gegangen ist. Und die Gründungsurkunde ist uns vollständig oder so gut wie vollständig erhalten. Sie bildet in unserer Aktensammlung mit den Auszügen der Abschiede zusammen ein Ganzes, das als eine Art literarischer Arbeit bezeichnet werden kann, und den Zweck hat die frischeingetretenen Regensburger über die bisher gefassten $\mathrm{Be}-$ schlüsse des Bundes und seine Grundlage ins Klare zu setzen, da es darauf ankam sie genau anzuweisen, zu was und wie sie im Einzelnen mitzuwirken verpflichtet waren. Man hătte ihnen die Abschiede einfach abschreiben können. So machte man es auch wirklich mit der Gründungsurkunde, die natürlich das Wichtigste war, und nur das Schlussdatum hat man da weggelassen. Anders verfuhr man mit den nachfolgenden Abschieden; wohl der Kürze wegen begnügte man sich damit, dem neuen Mitgliede das wichtigere und bleibendere daraus mitzutheilen. So entstand, die Gründungsurkunde an der Spitze, eine zusammenhängende Gedenkschrift über die bisherigen Bundesbeschlüsse, nicht immer sehr geschickt redigirt, aber doch so sehr sich an die ursprünglichen und wirklichen Abschiede auch in der Form anschliessend, dass man lange glauben konnte, man habe die authentischen Abschiede hier vor sich. Diese Gedenkschrift ist officiellen Ursprungs, darnach richtet sich der Grad und das Maass ihrer Glaubwürdigkeit. Ich habe diese kritischen Ergebnisse in meiner erwähnten Schrift ausführlich begründet.

Man sieht, dass ich, was das Rettungsgeschäft betrifft, in Betreff der Gründungsurkunde noch weiter gegangen bin als bei den darauf folgenden Abschieden. Ich habe darauf bestanden, dass sie uns weder als Entwurf noch als Auszug, sondern ganz oder fast ganz in ihrer authentischen Gestalt vorliege. Diess ist schon an sich wahrscheinlich, da gerade dieses Aktenstück für die Regensburger ebensosehr von grundlegender Bedeutung war wie für alle übrigen Bundesglieder. Die Gründungsurkunde mussten sie ganz haben, nicht in einem Auszug, am wenigsten in einem Entwurf. Wenn Busson $17 \mathrm{f}$. sie für das letztere hält, so ist der eine seiner Gründe auf ihre Form gestützt: sie ist nur von Städten ausgestellt, die Herren 
werden erst in Art. $2 a^{1}$ ) als Beigetretene erwähnt, diese Erwähnung der Herren erscheint als blosses Einschiebsel. Ich habe dagegen geltend gemacht, dass wir hier gar keine Gesammturkunde des Bundes sehen dürfen, welche dann allerdings von sämmtlichen Theilnehmern, nämlich Herren sowohl als Städten, ausgehen müsste. Vielmehr ist das von Anfang an nur die Urkunde der Stādte, welche für die Herren bestimmt war, und ihr entsprach ohne Zweifel früher eine ähnlich lautende andere Urkunde, welche von den Herren ausging und für die Städte bestimmt war. Herrenpartei und Stādtepartei des Bundes urkunden sich gegenseitig. Das entspricht ganz den Verhältnissen. Dass uns nur die städtische Urkunde erhalten ist, schliesst nicht aus, dass es frūher auch eine herrische gab, im Gegentheil, es weist darauf hin. Dass man für die Stadt Regensburg nur Eine der beiden abschrieb ist natürlich, weil sie Beide den gleichen Inhalt hatten; dass diess gerade die städtische war, ist auch natürlich, oder, wenn man will, zufällig. Ich habe da spātere Beispiele angeführt, wo auch Herren und Städte in āhnlicher Weise zusammentreten, so dass der Form nach nicht alle Bundesglieder, Herren und Städte, zusammen in einer einzigen Urkunde den Bund schliessen, sondern die Herren-Gruppe einerseits urkundet den Bund für die Städte-Gruppe andererseits, und umgekehrt, so dass ein untrennbares Zwillingspaar von Urkunden entsteht. Ich konnte dabei aber auch auf ein sehr naheliegendes Beispiel von Urkunden aus dem Jahr 1255 hinweisen. Damals nämlich haben Ende Juni Herren einerseits und Städte andererseits sich gegenseitig Urkunden ausgestellt, gleichlautend mutatis mutandis; wir haben davon keine mehr, aber sie sind Beide auf's Bestimmteste erwähnt in dem Schreiben der Stadt Mainz an die Westfălischen Städte vom 29. Juni 1255, bei Ennen-Eckertz Quellen zur Geschichte der Stadt Köln 2,355 Nr. 353. Da heisst es: In horum testimonium ac debitam firmitatem predicti domini ac nobiles presentis scripti tenorem nobis traditum sigillorum suorum munimine roborarunt, nosque ipsis vice versa idem scriptum sigillorum Maguntine, Wormaciensis et Spirensis omnium civitatum nomine tradidimus confirmatum ${ }^{2}$ ). Das Verfahren lässt Schrift.

1) Ich citire nach meiner neuen Ausgabe der Bundesakten in der erwãhnten

2) Der letztere Passus soll wohl heissen sigillorum Maguntine, Wormaciensis et Spirensis civitatum munimine omnium civitatum nomine tradidimus confirmatum. 
an Deutlichkeit nichts zu wünschen übrig. Sicher haben beide Urkunden einmal existirt, doch ist keine von ihnen wieder aufgefunden worden. Wohl aber hat sich die ein paar Tage nachher ausgefertigte Konfirmationsurkunde wieder gezeigt, dem Inhalt nach sicher identisch mil derjerigen, die durch sie konfirmirt wird, der Form nach auch wieder das eine Glied eines Urkundenpaares von Herren und von Stādten.

Diese Konfirmations-Urkunde gab mir den Anlass zu vorliegender kurzer Veröffentlichung. Sie kam mir erst zu, nachdem eben mein Buch gedruckt war. Durch die Zuvorkommenheit der Redaktion der Archivalischen Zeitschrift wird es mir möglich, sie so rasch nachzutragen. Sie ist eine Ergänzung zu meiner Schrift. Dr. Friedrich Ebrard in Strassburg machte mich darauf aufmerksam; es ist ein Stück aus dem Sponheimischen Archiv, das mit vielen andern Dingen bei der gegenseitigen Extradition von Archivalien, die auf Veranlassung Herrn von Löher's stattfand, aus dem französischen Departementalarchiv des Niederrheins an Bayern und in's Mūnchener Allgemeine Reichsarchiv kam, allerdings nur eine moderne Abschrift aus dem vorigen Jahrhundert, doch recht leidlich brauchbar. Ich halte es für sehr wohl möglich, dass auch das Original noch irgendwo existirt, vermuthlich in Karlsruhe, wo es noch 1779 vorhanden war, wie unsere Quellenangabe ausweist. Mit der Veröffentlichung wollte ich aber desshalb nicht warten. Der Redakteur der Archivalischen Zeitschrift, durch frühere Studien ${ }^{1}$ ) in diesem Stoffe völlig zu Hause, vermochte mehr als Einer den Werth dieses Diploms zu würdigen und so einem der vielen Kleinode seines Archirs den Weg in die Welt zu erschliessen. Für die Richtigstellung des Textes konnte das bereits erwāhnte Schreiben der Stadt Mainz vom 29. Juni 1255 herbeigezogen werden, das den Inhalt unserer Urkunde bereits kennt. So wird es möglich, zu einem, wie ich denke, sicheren Wortlaute zu gelangen.

Ich lasse hiemit die Urkunde folgen.

1) Franz Löher Fūrsten und Stādte zur Zeit der Hohenstaufen dargestellt an den Reichsgesetzen Kaiser Friedrich II. Halle 1846. 
Die Städtepartei des Rheinischen Bundes konfirmirt die Bestimmungen der Waffenruhe, welche sie zu Mainz am 30. Juni mit der Herrenpartei des Bundes bis 11. Nov. geschlossen hat. 1255, Juli 11, Bingen. $\begin{gathered}1255 \\ \text { Jul } 11 .\end{gathered}$

Nos Moguntinenses Colonienses Wormacienses Spirenses et alie civitates pacis federe ${ }^{21}$ conjuncte. noverint universi presencium inspectores, quod, cum quedam discordia super generalis pacis observatione inter nobiles terre ac civitates verteretur, utrique parti dies, videlicet proxima ante oclavam beati Johannis baptiste, ${ }^{b}$ in Moguncia est prefixa. in qua die nos in presencia domini Adolphi comitis ${ }_{J_{u n n}}^{12: 5}$. de Waldeck imperialis aule justiciarii super universis discordiis firmam pacem et treugas de consensu benivolo ${ }^{\text {c) }}$ partium ordinavimus usque ad festum beati Martini proxime ${ }^{\text {d) }}$ nunc venturum inviolabiliter observandas, confirmantes ipsam pacem et treugas mediante ${ }_{\text {Nov. } 11 .}^{1255}$ venerabili patre et domino nostro Gerhardo archiepiscopo Moguntinensi nobilibus ac nobis aput Pinguiam die dominica proxima ante festum beate Margarete, tali forma: [1] quod ipsi domini ac nobiles ${ }_{J u l .} 11$. medio tempore a bonis ecclesiarum cujuscunque fuerint ${ }^{\circ}$ religionis seu a bonis civium nullas omnino exactiones vel precarias aut alios ${ }^{\text {n }}$ census indebitos extorquebunt nisi tantummodo ea que scabini et villani qui huebenere vocantur sub juramento et banno ipsis dominis dictaverint de justicia esse danda [2] item villanos eorum pro eo quod cum civitatibus sibi vicinis pacem juraverint ${ }^{\text {s) }}$ in nullo penitus domini ${ }^{\text {b) }}$ molestabunt vel gravabunt; dicti vero villani dominis suis servicia, secundum" quod ipsis et antecessoribus suis ante 40 vel 50 annos facere consueverunt, facient. [3] item quicunque ex ipsis villanis medio tempore ad aliquam civitatum ${ }^{k}$ personali ${ }^{1 /}$ residencia voluerit se transferre, recipi poterit ab eadem jure civitatis per omnia fruiturus, ${ }^{\text {m) }}$ nec ex eo pax judicabitur violata. [4] si vero aliquis proprius dictas civitates ad manendum intraverit, domino ipsius eum

$M$ pace federis?

b) $M$ senkrechter Strich wher e.

c) $M$ benivola.

d) $\boldsymbol{M}$ proximo, $\boldsymbol{K}$ proxime.

e) $\boldsymbol{H}$ fuirint, $K$ fuerint.

ก om. $\boldsymbol{K}$.

g) $\boldsymbol{K}$ juraverant.

h) orn. $\boldsymbol{K}$.

1) $M$ sedm, $\boldsymbol{K}$ secundum.

k) $\boldsymbol{K}$ civitatem.

l) $K$ personali; in M hat p somohl das Zeichen für pre als das für per, sonst oie $K$.

m) $M$ frerviturus, $K$ fruitirus.

Archtvalische Zeltachrift. IV. 
requirenti secundum ${ }^{a)}$ jus et consuetudinem civitatum hucusque observatam per onnia satisfiet. [5] item cives, qui dicuntur palburgere, de cetero nullos habebimus. [6] item in omnibus et per omnia forma generalis pacis jurata in terra et in aquis ab universis conservabitur $^{\text {b) }}$ integra et illesa. [7] si vero medio tempore civitates

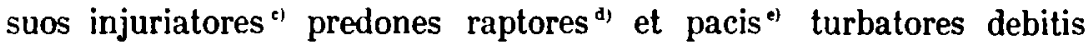
vindictis fuerint prosecuti, proinde pax minime censebitur violata. [8] item ad generalem pacem propagandam et confirmandam ${ }^{n}$ est ordinatum, quod dominus noster Wilhelmus rex Romanorum illustris in suo adventu ex ipsis dominis et nobilibus eliget ${ }^{8)}$ octo, quibus de universis civitatibus octo similiter ${ }^{\mathrm{h})}$ adjungentur: qui 16 mediante prefato domino nostro Wilhelmo rege ea, que generalis pacisi) propagationem et perpetuam confirmationem roborare ac perficere videbuntur, annuente divina gracia concorditer ordinabunt. quorum ordinationes et statuta tam domini quam civitates inviolabiliter observabunt. ad premissorum autem memoriam ac debitam firmitatem has litteras sigillo venerabilis patris domini nostri Gerhardi archiepiscopi Moguntinensis, Moguntinensium Wormaciensium et Spirensium, nomine omnium civitatum pacis federe conjunctarum, fecimus communiri. actum aput Pinguiam proxima dominica ante diem ${ }_{\text {Jul. } 11 .}^{1355}$ Margarete anno domini 1255.

$M$ aus Münch. $R$. A. Sponheim liasse 4 Nr. 2 (extradiert aus Depart. A. d. Niederrheins) cop. ch.; die Konformität der Abschrift mit ihrem Original, an dem 4 Siegel hiengen, ist unter Vordruckung des fürstl. geh. Kanzleisiegels bezeugt von J. G. Stösser, fürstl. markgr. bad. Hofrath und geh. Registrator, Karlsruhe, 13. Okt. 1779.

$K$ coll. die betr. Stellen des Schreibens der Stadt Mainz an Soest, Münster und andre ungen. Westfäl. Städte vom 29. Juni 1255 nach dem Abdruck aus dem Original des Köln. St.-A. in Ennen und Eckertz, Quellen zur Gesch. der St. Köln 2, 354 Nr. 353.

\footnotetext{
a) $M$ sedem, $K$ secundum.

b) $K$ ald medio tempore.

c) $M$ injuritatores, $K$ injuriatorea.

d) oon. $K$.

c) $\boldsymbol{M}$ pacem.

f) $K$ om. p. e. c., folgt de consensu parcium est statutum quod - .

g) $M$ eligert, $K$ eliget.

h) om. $K$.

i) $\boldsymbol{K}$ generalem paccm, om. das folgende p. e. p. c.

k) $K$ adil. per omuia, das vielleiche das folgende hier fehlende t. d. q. c. ersetzen soll.
} 
Diese Urkunde kommt in der schon erwähnten Sammlung der Bundesakten gar nicht vor, auch der Tag von Bingen nicht auf dem sie entstand. Aber das darf uns nicht misstrauisch gegen unsre Aktensammlung machen. In unsrer Urkunde handelt es sich nur um etwas vorübergehendes. Es hatte Streitigkeiten gegeben zwischen Herren und Städten des Bundes, man lernt sie aus unserem Aktenstücke kennen, das bestimmt ist, sie vorläufig beizulegen; ein Waffenstillstand kommt bis 11. Nov. 1255 zu Stande, unser Aktenstück enthält die Normen, nach denen man sich in den streitgen Punkten bis dahin richten soll. $\mathrm{Zu}$ der Zeit nun, wo die Bundesakten zusammengestellt wurden ${ }^{1}$, Anfang Okt. 1256, war dieser Waffenstillstand längst abgelaufen, und die Kenntniss seiner Bestimmungen daher für ein neu eintrelendes Mitglied, wie die Stadt Regensburg, nicht mehr nothwendig, deshalb liess man sie aus der Sammlung weg, die man den Regensburger Boten mit nach Hause gab. Ebenso fehlt ja mit Recht in jenen Akten auch der Inhalt der beiden Waffenstillstandsurkunden, der in dem Briefe vom 29. Juni 1255 angeführt wird.

Wie verhālt sich nun aber unsre Urkunde vom 11. Juli, zu der man als zu der stādtischen sich eine gleichlautende herrische zu denken hat, zu diesen beiden Waffenstillstandsurkunden vom Ende Juni? Vom Waffenstillstand handelt es sich das einemal wie das andremal. In unsrer Urkunde heisst es in Eingang: confirmantes ipsam pacem et treugas - tali forma. Ebenda heisst es von den vorausgegangenen Mainzer Urkunden: super universis discordiis firmam pacem et treugas - ordinavimus, und entsprechend in jenem Briefe vom 29. Juni: pacem et treugas - ordinatas esse in hunc modum, und in dem Brief der Städte an den König vom 30. Juni abermals: firma pax et treuge stabiles super universis guerris ${ }^{2}$ ) et discordiis sunt statute. Auf dem Mainzer Bundestag vom 29. Juni war also in der That die Waffenruhe oder der Waffenstillstand mit seinen Interimsbestimmungen bereits festgestellt, die Urkunden darūber waren zwischen Herren und Städten auch gleich ausgetauscht

') Siehe meine Scbrift S. 96.

2) Die Waffen waren also wirklich schon gebraucht worden, und darnach muss die Behauptung mejner Schrift S. 106 verändert werden, wo kein eigentlicher Kriegszustand zwischen Herren und Stădten angenommen wird. Ein grosser und allgemeiner Krieg war es wohl nicht, aber verschiedene Fehde-Zustãnde. 
worden (traditum, tradidimus, in dem Brief). Die Bingener Urkunden vom 11. Juli ordnen also denselben Gegenstand wie die Mainzer vom Ende Juni. Sie ordnen ihn aber auch ganz in der gleichen Weise. Das letztere ergiebt eine genaue Vergleichung des Bingener Aktenstücks mit dem oftgenannten Briefe, der den Inhalt der Mainzer Abmachungen an die Westfâlischen Städte mittheilt. Der Brief stimmt fast wörtlich mit unsrer Urkunde, dem Sinne nach ganz, die Abweichungen sind lediglich stilistische, und nichts steht in dem einen Stück, was nicht auch in dem andern stünde. Die Bingener Urkunde ist also wirklich nichts andres, als das, wofür sie sich ausgiebt: eine Konfirmation der Mainzer Abmachungen; sconfirmantes« sagt sie ja selbst. Auch hat sich der Mainzer Brief an die stādtische Ausfertigung gelialten, man sieht das aus den Worten: item cives, qui dicuntur palburgere, de cetero nullos habebimus; und unsere Urkunde ist ebenfalls eine stādtische; selbst darin sind sie sich gleich, obschon das nur ein gleichgiltiger Zufall ist.

Es entsteht aber die Frage: warum, wenn auf den beiden Tagen zu Mainz und zu Bingen ganz das gleiche geschah, warum ist es denn zu Bingen noch einmal geschehen, nachdem es vor nicht ganz zwei Wochen schon zu Mainz geschehen war? Die Urkunde gibt wohl einige Auskunft in ihrem Eingang. Da heisst es, dass die beiden Parteien zu Mainz ihre Waffenruhe festgestellt haben in presencia domini Adolphi comitis de Waldeck imperialis aule justiciarii, und dass sie dieselbe konfirmirt haben zu Bingen mediante venerabili patre et domino nostro Gerhardo archiepiscopo Moguntinensi. Die Feststellung also geschah in Gegenwart des königlichen Stellvertreters, die Konfirmation unter Vermittlung des Erzbischofs von Mainz. Für die letztere wurde eine neue Versammlung, von der man bisher gar nichts wusste, in dem Kurmainzischen Orte Bingen gehalten, der Zeit nach so hart an dem eben gehaltenen Mainzer Bundestag, dass man versucht ist, sie als die blosse Fortsetzung des letzteren zu betrachten. Es liegt nun nahe, aus dieser āusseren Verschiedenheit beider Vorgänge den Grund zu erschliessen, warum überhaupt eine zweite Versammlung und eine Konfirmation des bereits Festgestellten für nöthig erachtet wurde. Wie in Bingen der königliche Stellvertreter nicht mehr anwesend war, so hatte sich offenbar zu Mainz der Erzbischof nicht eingefunden. Das Erscheinen des Einen muss das des Andern ausgeschlossen haben. Wenn das, was Beide thun, dasselhe ist, so kam es offenbar nicht auf den In- 
halt dieses Thuns an, sondern auf die Form unter der es geschehen sollte. Die Sache ist, dass Beide nicht zusammen handeln, wcil jeder für sich handeln will, um die hervorragende Stellung des Andern nicht anzuerkennen. Waldeck kann auf sein Recht nicht verzichten, in der Bundesversammlung als Stellvertreter des Königs zu wirken; andrerseits will der Kurfürst, als das vornehnste Mitglied der Herrenpartei, die leitende Vermittlerrolle spielen, und manche Herren mögen ihm darin zugestimmt haben. Waldeck kann für sich wesentlich auch nichts anderes beansprucht haben als diese Vermittlerrolle, diese aber jedenfalls, und deswegen erschien der Erzbischof, wohl sammt seinem Anhang, zu Mainz nicht, wo Waldeck auftrat. Da jedoch andre Herren und besonders die Städte keinen Anstoss an Waldeck nahmen, so wurde die Sache wirklich schon zu Mainz unter seiner Führung abgemacht; es wird Theil genommen haben wer hier anwesend war; Mainz, Worms und Speier besiegeln die Urkunde im Namen aller Städte (s. das Ende jenes Briefs). Man muss von da aber auch noch nach Bingen gegangen sein, um dem Erzbischof den Gefallen zu thun und ihn zu gewinnen. Da findet denn die Konfirmation statt, wieder siegeln Mainz, Worms und Speier im Namen aller verbündeten Stādte, ausserdem aber auch der Erzbischof, und wie er hier unsere städtische Urkunde mitsiegelt, so wurde es ohne Zweifel auch bei der herrischen gehalten, während Waldeck das nicht gethan hatte. Es wird eben nachträglich jedes Mittel aufgewendet, um den Kurfürsten an erster Stelle erscheinen zu lassen. Daher sagt unsere Urkunde von Waldeck nur, dass er in Mainz anwesend war (in presencia), auch im Mainzer Abschied III 1 wird es nur so ausgedrückt (presente) ${ }^{1}$ ). In Bingen erst, so soll es erscheinen, hat der Kurfürst wirksam vermittelt, mediante sagt daher unsere Urkunde von ihm, recht im Gegensatz zu dem, was sie von Waldeck zu berichten weiss. Das ist nun gewiss nicht die richtige Darstellung der Sache. Waldeck hat in Mainz sicher den Vermittler gemacht. Was hätte er denn sonst thun sollen? In der That sprechen das die Städte in ihrem Brief an den König, wo sie nicht auf den Erzbischof Rücksicht zu nehmen hatten, auch offen aus: mediante nobili viro de Waldechen imperialis aule justiciario (Bölımer l. c. 95). Das mediante aber bezeichnet eben den leitenden

1) In dem oft erwăhnten Brief an die Westfālischen Stădte wird sein Name nicht genannt. 
Einfluss, wie in Art. 8 unsrer Urkunde mediante-rege. Der Erzbischof hatte also in Bingen nichts mehr zu vermitteln, faktisch war ja schon in Mainz alles vermittelt worden, nur durch einen Andern. Doch der Kurfürst wollte und sollte eben als die leitende Stelle erscheinen, und zwar er allein, und so wurde es nun in unserer Urkunde dargestellt für Jeden, der es glauben mochte. Thatsächlich blieb ihm in Bingen weiter nichts mehr übrig, als nachtrăglich mit seinem Anhang beizutreten ${ }^{1}$ ), und nur dies kann der Inhalt des Bingener Tags gewesen sein. Es kann zunächst als ein blosser Wort- und Rangstreit erscheinen. Aber es ist mehr als dies. Man erblickt hier den eifersüchtigen Erzbischof in offner Opposition gegen den königlichen Justitiar, der an Stelle des Königs auftritt. Opponirt er aber gegen des Königs Stellvertreter, so opponirt er auch gegen den König. Soviel ist deutlich an der Sache. Das auffallend dringliche Verlangen der Städte nach der Ankunft des Königs, das sic in ihrem Brief an diesen aussprechen (Böhmer l. c. 95), hängt gewiss damit zusarnmen. Ob Gerhard etwa selbst ein Recht beanspruchte, den abwesenden König zu vertreten, sieht man nicht. Ich habe aber schon früher ${ }^{2}$ ) ausgesprochen, dass die Eindrängung des Königs und seines Justitiars als richterlicher Bundesbehörde Widerspruch gefunden zu haben scheine, und zwar gleich Anfangs im Frühjahr 1255. Das wird nun sehr angenehm bestätigt, wenn der Justitiar sogleich bei seiner ersten Anwesenheit auf einem Bundestag eine solche Opposition gegen seine Wirksamkeit bei dem vornehmsten Fürsten des Bundes findet. Alles stimmt. Vermuthungen über weitere Zusammenhänge unterlasse ich. Der Werth unserer Urkunde tritt schon durch diese selbstverständlichen Ergebnisse in helles Licht.

Ich habe in meiner Schrift S. 74 den erwähnten Brief an die Westfälischen Städte unrichtig für einen Auszug des Mainzer Abschieds vom Ende Juni 1255 angesehen, da ich unsere Urkunde noch nicht kannte. Jetzt sieht man, dass jener Brief sich nur mit den Bestimmungen der Waffenruhe beschäftigt, welche in den verlorenen Zwillingsurkunden des Mainzer Tags niedergelegt waren, und

1) Die Stadt Mainz berichtet in dem erwähnten Brief auch gleich von der Mainzer Versamnlung aus an die Westfălischen Stãdte den Abschluss der Waffenruhe als etwas vōllig Fertiges und Abgemachtes.

$\left.{ }^{2}\right)$ Siehe meine Schrift S. 211. 
dass es neben diesen Urkunden noch eiuen ganz selbständigen Abschied gab, dessen Auszug die Nr. III. meines Abdrucks ist ${ }^{1}$ ). Dieser Auszug hat nur zwei Artikel. Der erste davon, über die Judenzinse, ist ihm ganz eigenthümlich. Der zweite wiederholt zwar aus den Urkunden der Waffenruhe die Aufhebung des Pfahlbürgerthurns, aber doch nur um nähere Ausführungsbestimmungen daran zu knüpfen, die in den Urkunden nicht standen. Ob und welche weitere Artikel der unverkürzte Abschied enthalten hat, ist nicht zu sagen; wahrscheinlich nicht diejenigen, welche schon in den Urkunden standen.

Noch eine andere Frage wird durch unsere Urkunde wie durch den erwähnten Brief beleuchtet. Man kann freilich schon ohnedies sagen, dass für das Interim bis 11 . Nov. nicht etwa die Bestimmungen der Waffenruhe an die Stelle der Bundesbestimmungen getreten sind, sondern dass auch während dieser Waffenruhe die letzteren und der Bund selbst als fortdauernd betrachtet werden. Sonst gābe es schon von dem Mainzer Tag, Ende Juni, keinen Bundesabschied Nr. III., und ebenso könnte im August kein Bundesabschied Nr. IV. und im Oktober kein Bundesabschied Nr. V. erwartet werden, die doch da sind ${ }^{2}$ ). Diese Fortdauer des Bundes selbst wird nun nicht bloss in jenem Brief an die Westfälischen Städte, sondern fast ganz gleichlautend auch in unserer Urkunde ausgesprochen: item in omnibus et per omnia forma generalis pacis jurata in terra et in aquis ab universis conservabitur (der Brief setzt hinzu: medio tempore) integra et illesa. Die forma generalis pacis ist nämlich die Gründungsurkunde vom Juli 1254. Zum leichteren Verständniss unserer Urkunde füge ich meine Beobachtungen über deren Sprachgebrauch hinzu, weil pax in ihr bald den Bund, bald die Waffenruhe bedeutet. Man muss also sorgfältig scheiden. Der Bund selbst ist in unserer Urkunde zu verstehen unter pacis federe in der Inskriptio, unter generalis pacis in der darauf folgenden Einleitung, unter pacem in Art. 2, unter pax in Art. 3, unter forma generalis pacis in Art. 6, unter pacis turbatores und pax in Art. 7, unter ad generalem pacem propagandam und generalis pacis propagationem in Art. 8, unter pacis federe in der Siegelankündigung;

') Auf S. 23 f. meiner Schrift.

2) Ich zăhle die Abschiede nach den Ziffern, mit denen ich sie in meiner Schrift edirt habe. 
auch in pacis turbatores im Art. 7 ist das Wort pax als der Bund zu verstehen, wenn nicht im allgemeinen Sinn (Friedensstörer). Dagegen firmam pacem et treugas und ipsam pacem et treugas in der Einleitung bedeutet die Waffenruhe. Ganz ebenso ist der Sprachgebrauch in dem öfter angeführten Briefe bei Ennen-Eckertz 2 Nr. 353. In dem Brief der Stādte an den König vom 30. Juni 1255 (Böhmer 95) ist firma pax et treuge stabiles die Waffenruhe, das pacem terre salubriter inchoatam bezeichnet den Bund. Zum Verständniss dieser drei Stūcke ist es wichtig, diesen Sprachgebrauch festzustellen. Der Abschied unseres Tags, in meiner Edition Nr. III, hat diese Ausdrücke gar nicht, und ausser diesen vier Stücken haben wir nichts von diesem Tag.

Busson 55 sieht ganz recht, dass die Streitigkeiten zwischen der Herrenpartei und der Städtepartei des Bundes ihren Grund hauptsāchlich in dem Verhältniss der Städte zu der Bauernschaft hatten, und im Zusammenhang damit steht auch das Pfahlbürgerthum. Diese Dinge stehen auch in unserer Urkunde im Vordergrund. Ich kann sie hier nicht näher erörtern, sie wären Gegenstand einer eigenen Untersuchung. Gewiss sind aber in Art. 8 unserer Urkunde unter den Anordnungen, welche bei Ankunft des Königs der von ihm eingesetzte gemischte Sechzehner-Ausschuss treffen soll, auch solche verstanden, welche von jenen Verhältnissen handeln sollten, etwa dass die vorübergehenden Bestimmungen des Interims zu bleibenden gemacht würden, mit oder ohne Veränderung. Von dieser Kommission nun hört man weiter nichts. Man kann jetzt, seit wir die neue Urkunde haben, nur sagen, dass ich nicht Recht hatte, zu meinen, die Stădte hătten ihre acht Vertreter zu diesem Ausschusse selbst bezeichnen dürfen, während die acht Vertreter der Herren vom König ausgewählt wurden. Denn es heisst in unsrer Urkunde Art. 8: quibus de universis civitatibus octo similiter adjungentur; das similiter, das uns bisher fehlte, zeigt, dass die Bestellung der Ausschussmitglieder bei den Städten dieselbe war wie bei den Herren, also ebenfalls der Auswahl des Königs vorbehalten blieb. Von dieser Kommission aber verlautet also fernerhin nichts mehr. Sie wird wohl in Wirksamkeit getreten sein, aber ohne etwas zu Stande zu bringen. Die von den Städten (Böhmer 95) gewünschte Konfirmation des Bundes giebt der König zwar, bei Ablauf des Interims, feierlich am 10. Nov. 1255 zu Oppenheim (Böhmer 95 f.), aber sie enthält nichts über das Verhältniss der Städte zu der Bauernschaft und 
nichts über das Pfahlbürgerthum. Jeder, heisst es, soll sein Recht behalten. Die Frage aber war, was dieses Recht sei. Indem man das überging, verzichtete man auf die Regelung dieser Verhältnisse. Dem König war die Sache offenbar zu schwer, er mochte es mit Niemand verderben, und er konnte das wohl auch nicht; es war einfach und bequem, dass Jeder sein Recht behalten sollte. Warum man nicht einfach diejenige Regelung bleibend machte, die durch das Interim fixirt war, ist schwer zu sagen. Vielleicht, dass den Herren die Ausführungsbestimmungen über das Pfahlbürgerthum in dem Mainzer Abschied III, 2 nicht gefielen; anderes wird hinzugekommen sein. Man kann aber sagen, dass mit dem Verzicht auf die Ordnung aller dieser Verhältnisse schon allein der aus Herren und Stādten zusammengesetzte Bund zum Untergang verurtheilt war, wenn auch sonst nichts hinzugekommen wäre. 